\title{
Intraductal Papillary Carcinoma of the Breast: A Case Presentation
}

\section{Carcinoma Papilar Intraductal de Mama. Pre- sentación de un Caso}

Luisa Paulina Chafla Romero1, Diana Belén Cuenca Mora ${ }^{2}$, Nidia Narcisa Auqui Calle ${ }^{3}$, María Romyna Delli Villavicencio ${ }^{2}$, and Felipe Antonio Larrea Lara $^{2}$

${ }^{1}$ Escuela Superior Politécnica de Chimborazo, Ministerio de Salud Pública del Ecuador, Centro de Salud Yaruquíes, Riobamba, Ecuador

XVIII International Seminar on Health, Food and Human Nutrition

Corresponding Author:

Luisa Paulina Chafla Romero pauly_mariuxi@hotmail

Published: 9 September 2021

Production and Hosting by Knowledge E

(c) Luisa Paulina Chafla Romero et al. This article is distributed under the terms of the Creative Commons Attribution License, which permits unrestricted use and redistribution provided that the original author and source are credited.

G OPEN ACCESS

${ }^{2}$ Unidad Oncológica Solca, Riobamba, Chimborazo, Ecuador

${ }^{3}$ Ministerio de Salud Pública, Centro de Salud Santa Rosa, Riobamba, Chimborazo, Ecuador

\section{Abstract}

Intraductal papillary carcinoma, also known as papillary ductal carcinoma in situ, forms part of a heterogeneous group of breast lesions, the reported incidence rates are very low and occurs in $27.7 / 100,000$ in women, although the percentage of mortality is only $1 \%$. This carcinoma is characterized because it can affect the inside portion of the mammary ducts, respecting the basement membrane, with proliferation of neoplasic cells, and since it is not in contact with the lymphatic vessels the possibility of metastasis is almost null. However, the risk of progressing to invasive cancer is nevertheless multifactorial as well as its size, histology, and resection margin. Clinically, it can be characterized by the presence of a single or multiple mass of moderate size, and on a mammograph it's seen as nodular lesions with irregular edges. The case of an older adult female patient with a mammary nodule with 5 months of evolution, until it forms into a mass and later the execution of routine and laboratory examinations, allowing the diagnosis of intraductal papillary carcinoma to be confirmed. Due to the complexity of the clinical presentation and the age of the patient, a radical mastectomy and physiotherapy of the right upper limb was performed. intraductal papillary carcinoma represents less than $1 \%$ of all mammary carcinomas, and has a good prognosis.

Keywords: carcinoma, mom, papillary.

\section{Resumen}

El carcinoma papilar intraductal, también conocido como carcinoma ductal in situ papilar, forma parte de un grupo heterogéneo de lesiones de la mama, la tasa de incidencia es muy baja y se presenta en $27,7 / 100.000$ en mujeres, aunque el porcentaje de mortalidad es de tan solo el $1 \%$. Se caracteriza porque en el interior de los ductos mamarios y respetando la membrana basal hay proliferación de células neoplasias, y al no encontrarse en contacto con los vasos linfáticos la posibilidad de metástasis es casi nula, más sin embargo el riesgo de progresar a cáncer invasor es multifactorial como su tamaño su histología y el margen de resección. Clínicamente se caracteriza por ser masas únicas o múltiples de tamaño moderado y en la mamografía se observan como lesiones nodulares de bordes irregulares. Se presenta el caso de una paciente adulta mayor que presenta un nódulo mamario de 5 meses de evolución, hasta tornarse en una masa y posterior a la ejecución de exámenes de gabinete y de laboratorio se confirma el 
diagnóstico de carcinoma papilar intraductal, por la complejidad del cuadro y por la edad de la paciente, se realiza como tratamiento mastectomía radical y fisioterapia del miembro superior derecho. El carcinoma papilar intraductal representa menos del $1 \%$ de todos los carcinomas mamarios, y es de buen pronóstico.

Palabras Clave: carcinoma, mama, papilar.

\section{Introducción}

El cáncer de mama, es la primera causa de muerte en las mujeres a nivel mundial, la incidencia se incrementó de manera vertiginosa, en los países en vías de desarrollo como el Ecuador, la taza de mortalidad es de por cada 13,2 por cada 100 mil féminas, el diagnóstico se realiza en etapas terminales, como consecuencia de los pocos recursos económicos que destinan los estados, para la detección oportuna del cáncer mamario.

Es de vital importancia para el diagnóstico oportuno y el tratamiento adecuado, conocer diversos aspectos de la patología mamaria como la clasificación histológica del cáncer de mama (CM) que ha posibilitado determinar dos distintos grupos: $\mathrm{El}$ carcinoma in situ y el carcinoma invasor $(\mathrm{Cl})$. El $\mathrm{Cl}$ es aquel que invade más allá de la membrana basal y se introduce en el estroma mamario donde puede llegar hasta los vasos sanguíneos, ganglios linfáticos regionales y causar metástasis a distancia. Entre sus principales tipos histológicos se encuentran el carcinoma ductal, lobulillar, tubular, mucinoso, medular, papilar y el metaplásico [1, 2].

Las principales manifestaciones del carcinoma es la presencia de secreción clara o sanguino lenta por el pezón y la existencia de una masa que se encuentran en lesiones periféricas en forma de microcalcificaciones [3]. Este tipo de carcinoma se manifiesta de forma frecuente en la edad adulta [4]. El carcinoma papilar sólido de mama, una entidad recientemente definida, es poco reconocido, y su naturaleza y manejo aún se debaten $[5,6]$. Este grupo de tumores se localiza y extiende exclusivamente en el sistema ductal mamario, en cualquier sitio desde el pezón hasta la unidad ductolobular terminal, y comparten una característica histológica en común: La proliferación del epitelio mamario soportada por un tallo fibrovascular [7, 8].

El diagnóstico del carcinoma papilar intraductal presentó una notable evolución con la introducción de la mamografía, pues con la utilización de este medio, el diagnóstico es temprano y a gran escala, porque se detecta la neoplasia en etapa no palpable [9].

Dentro del punto de vista macroscópico no se encuentra una característica específica. Dentro del punto de vista microscópico se observan conductos o unidades de conductos en los que se encuentran tallos fibrosos y vasculares delgados a los que cubren un conjunto de células epiteliales neoplásicas [10].

El carcinoma papilar intraductal teóricamente tiene una baja mortalidad, pero la finalidad es disminuir la posibilidad de recidiva, las opciones terapéuticas se presentan desde la mastectomía total, hasta la conservación de la mama con la resección del 
tumor la misma que se realiza de forma conjunta con la administración de radioterapia y terapia hormonal [11].

El estudio del siguiente caso clínico, tiene como objetivo conocer las manifestaciones clínicas, diagnóstico y tratamiento de un tipo de cáncer mamario de baja incidencia pero de buen pronóstico.

\section{Caso}

\subsection{Información del paciente}

Mujer de 91 años de edad, nacida y residente en Quito, instrucción superior completa, estado civil unión libre, católica, jubilada. Grupo sanguíneo: O Rh+ Transfusiones hace 40 años.

Antecedentes patológicos personales: Hipertensión arterial + artrosis de cadera.

Antecedentes patológicos quirúrgicos: Colecistectomía hace 20 años, resección intestinal hace 40 años, histerectomía.

Hace 20 años, resección de lipoma en mama derecha hace 40 años.

Antecedentes patológicos familiares: Madre de la paciente presenta Diabetes Mellitus tipo 2, e hipertensión arterial.

Antecedentes Gineco obstétricos G: 5 P: 5 A: 0 C: 0 Hijos vivos: 4

Métodos anticonceptivos: No refiere uso de métodos hormonales.

Telarquia a los 12 años, menarquia: 14 años, menopausia: 58 años, paptest hace 5 años: El mismo que no se encontró patología.

Medicamentos: Losartán 100 mg cada día, parches de Buprenorfina cada lunes y jueves.

Hábitos: Alimenticio: 3 veces al día (balanceada). Miccional: 4 veces al día - defecatorio: 2 diario.

Tabaco: Niega - alcohol: Niega - drogas: Niega.

\subsection{Hallazgos clínicos}

\subsubsection{Examen físico general}

TA: 130/74 FC: 68 x FR: 18 Sat O2: 83\% Peso: 79,3 kg Tallas: 1,44, paciente consciente, orientado, hidratado, afebril en decúbito dorsal activo, colaborador.

\subsubsection{Exámen físico regional}

(i) Cabeza: Normocefálica, cabello entrecano de implantación normal

- Ojos: Pupilas normoreactivas a la luz y acomodación, conjuntivas rosadas, escleras anictéricos. 
- Oídos: Conducto auditivo externo permeable.

- Nariz: Fosas nasales permeables con buena entrada de aire bilateral.

- Boca: Mucosas orales húmedas, orofaringe no congestiva.

\section{(ii) Cuello}

No adenopatías, tiroides no palpable no visible no masas.

\section{(iii) Tórax}

Simétricos expansibilidad conservada. Mama derecha se palpa masa multilobulada blanda de aproximadamente $5 \mathrm{~cm}$ bordes no definidos, no dolorosa a la palpación, complejo areola pezón conservado bilaterales, no se observan cambios en la piel, no se palpan adenopatías axilares.

- Pulmones: Murmullo vesicular presentes.

- Corazón: Rítmico, regular, no soplos.

\section{(iv) Abdomen}

Blando, depresible, no doloroso a la palpación, no se palpan visceromegalia.

\section{(v) Extremidades}

Dolor a la movilización en articulación coxofemoral derecha no edemas. Control neurovascular distal normal.

\subsection{Time line/línea cronológica}

Paciente de 91 años refiere que hace 5 años acude para realización de mamografía y ecografía por palpación de nódulo en mama derecha con resultados negativos, desde hace 5 meses paciente evidencia crecimiento de masa por lo que se realiza mamografía bilateral

22 de agosto 2019. Se realiza mamografía.

25 de septiembre del 2019. Se realiza eco mamario.

29 de noviembre del 2019. Se realiza core biopsia.

13 de enero del 2020. Se realiza Tomografía axial computarizada de cuello y tórax.

27 de enero del 2020. La paciente es sometida a mastectomía radical, con disección ganglionar axilar la misma que se desarrollo sin complicación aún tomando en consideración la edad de la paciente. Se solicita histopatológico de la pieza quirúrgica. 
30 de enero 2020 se envía a domicilio con medicamentos para sus enfermedades crónicas degenerativas y se prescribe 4 días más de antibiótico terapia y 5 días para tratamiento analgésico.

7 de febrero se realiza control y retiro de dren la paciente manifiesta intenso dolor en miembro superior derecho por lo que se continua con analgesia.

14 de febrero se recibe histopatológico cuyo informe confirma el diagnóstico antes descrito.

18 de marzo la paciente acude a control manifiesta dolor y paresias en la zona de herida quirúrgica fue enviada a fisioterapia del miembro superior derecho.

\subsection{Evaluación diagnóstica}

El diagnóstico fue dado por la ecografía que observa una lesión sospechosa en el cuadrante inferior externo (Figura 2), por que se procede a la ejecución de una core biopsia con guía ecográfica (Figura 3) de la que se obtiene 10 fragmentos los mismos que posterior al estudio anatomopatológico se evidencian papilas frondas de tejido fibroganglionar revestidas de células tumorales, y posterior a la clínica y exámenes de gabinete y laboratorio se llega al diagnóstico de Carcinoma Papilar intraductal.

Mamografía (Figura 1) se valora mamograma, no existen estudios previos. Las mamas son asimétricas en tamaño con predominancia izq. Patrón radiológico de tipo fibroglandular disperso.

En mama derecha. CE región mamaria profunda lesión multilobulada compuesta de 5 lesiones nodulares de bordes no circunscritos que fluctúan entre 7 y $24 \mathrm{~mm}$ de diámetro densas es necesario ecografía. Calcificaciones de tipo benignas bilaterales. Dilatación venosa bilateral con predominio derecha. Piel y pezones normales. Tejido mamario ectópico y ganglios axilares bilaterales.

\section{Conclusión}

BIRADS 0. Valoración incompleta.

Es necesario ecografía mamaria.

Ecografía de mama (Figura 2). En mama derecha hacia el CIE quiste complejo con lesión interna irregular sólida la masa quística compleja mide $51 \times 23 \mathrm{~mm}$, con lesión sólida interna irregular de $24 \times 16 \mathrm{~mm}$, vascularización interna. Dilatación venosa cuadrante superior externa ya diagnosticada mamográficamente que llega hasta 4 $\mathrm{mm}$. de grosor. En mama izquierda no se observan lesiones sólidas ni quísticas intraparenquimatosas. No adenopatías axilares. Lesión sospechosa en mama derecha CIE a descartar papilomatosis múltiple. BIRADS IV.

Core biopsia 28 de noviembre del 2019 (Figura 3). Se procede a realizar core biopsia por guía ecográfica sobre lesión en mama derecha cuadrante inferior externo. Se obtienen ocho fragmentos los cuales se envían al departamento de patología. Se drena 10 cc de líquido cero hemático. 


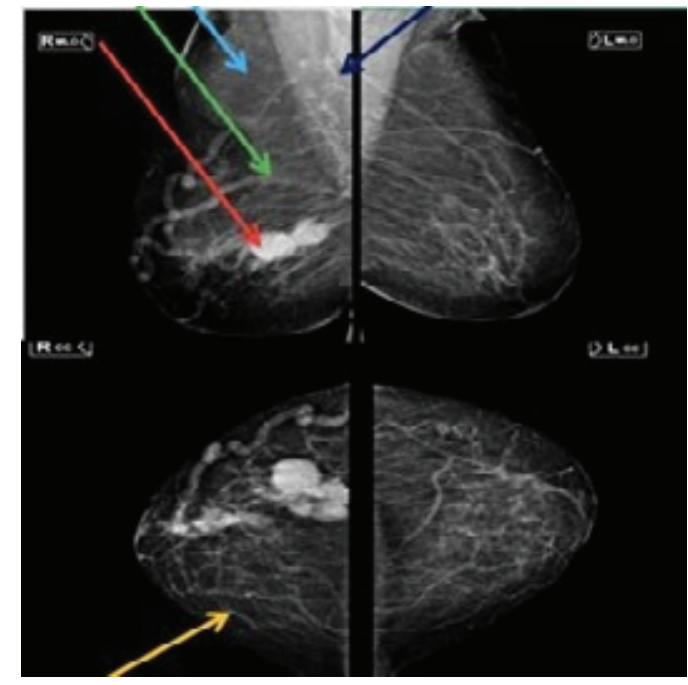

Figure 1

Mamografía, lesión multilobulada compuesta de 5 nódulos de bordes no circunscritos.
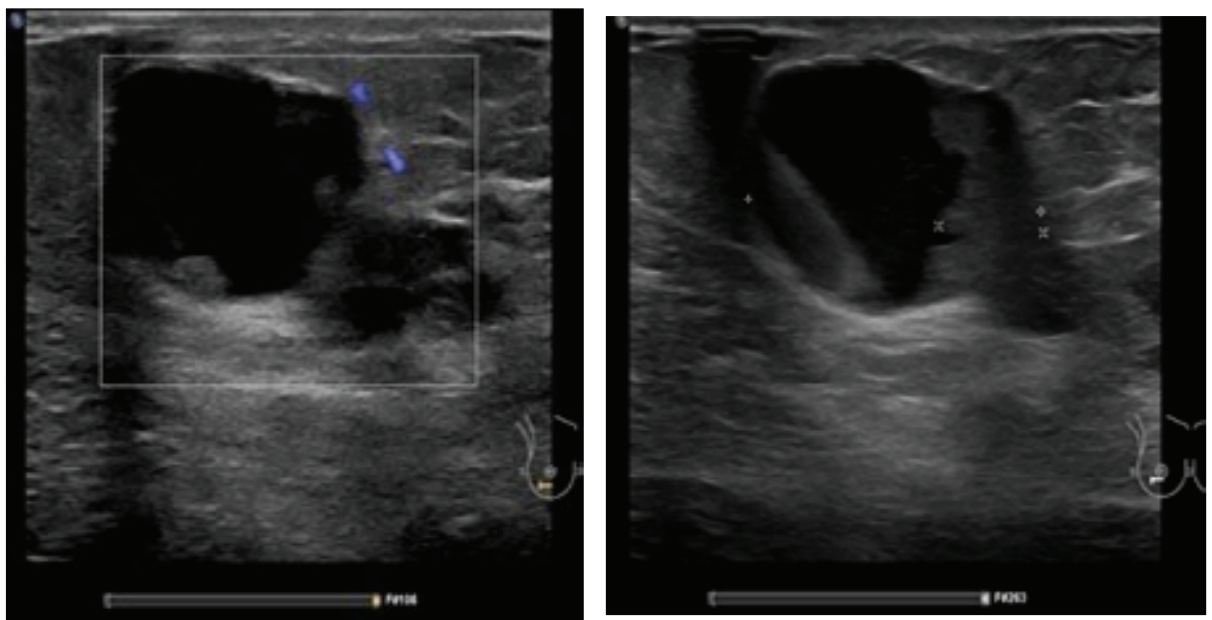

Figure 2

Ecografía de mama. Masa sólida quística compleja con lesión sólida irregular.

Tc simple y contrastada de cuello. Estructuras glandulares del cuello sin alteraciones patológicas. Vía aérea superior central y permeable. Ganglios hipodensos, ovalados cervicales bilaterales con diámetros cortos de $5 \mathrm{~mm}$ no significativos oncológicamente. Cambios degenerativos difusos en columna cervical.

Tc de tórax. En la mama derecha y hacia los cuadrantes externos masa tumoral de densidad mixta, sólida quística, mal definida, de contornos bilobulados, de componente sólido capta heterogéneamente el medio de contraste, a través de finos septos interconecta con otras pseudolesiones sólidas definidas, contornos espiculados, captantes de contraste, en número de dos que se proyectan anteriores a la lesión antes mencionada y mide $32,1 \times 17,0$ y $25,3 \times 19,2 \mathrm{~mm}$, en tanto que la lesión de mayor tamaño mide $49,9 \times 25,0 \mathrm{~mm}$. 

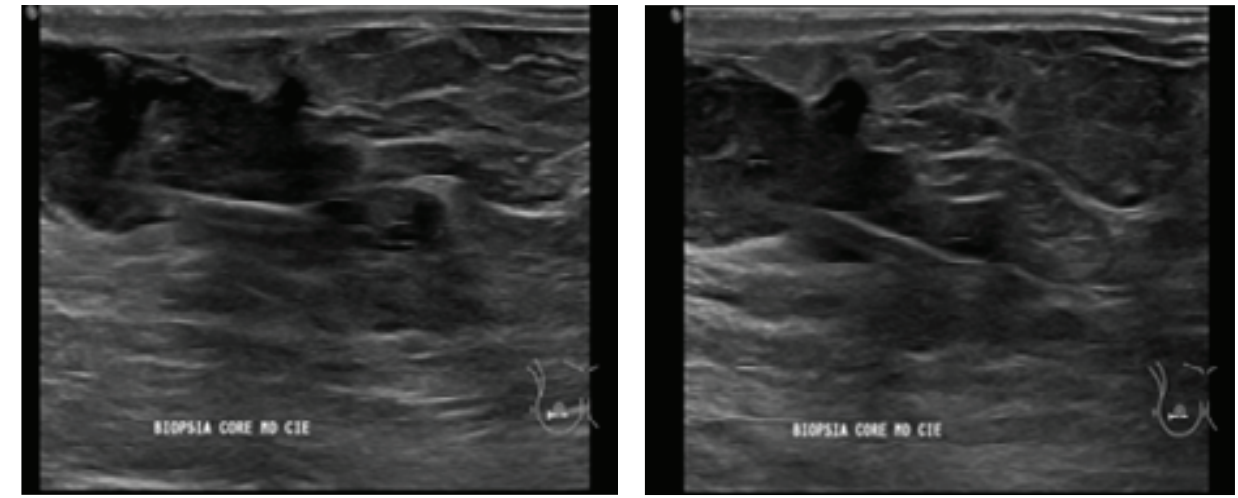

Figure 3

Core biopsia por guía ecográfica, sobre lesión en mama derecha.

Respeta los planos musculares profundos, asocian engrosamiento y retracción de la piel adyacente, distorsionan al tejido graso aledaño. En la región axilar de este mismo lado dos ganglios redondeados y levemente captantes de contraste con diámetros cortos de hasta $8,5 \mathrm{~mm}$. Estructuras vasculares dilatadas y tortuosas en la región axilar derecha y porción externa de la mama de este mismo lado.

Glándula mamaria izquierda dentro de parámetros normales para la edad.

Ateromas calcificados en el cayado aórtico, aorta descendente y vasos coronarios. Área cardíaca incrementada de tamaño.

Tejidos blandos de la pared torácica sin alteraciones patológicas.

Trama broncovascular bilateral discreta y difusamente engrosada, no se identifican consolidaciones ni derrame pleural. En el pulmón derecho segmento III tenue pseudonódulo periférico, no calcificado, no bien definido de $4 \mathrm{~mm}$.

Moderados cambios degenerativos difusos en columna dorsal Ca. de mama derecha ya conocida, asocia ganglios metastásicos axilares ispilaterales.

\subsection{Intervención terapéutica}

La paciente fue sometida a una mastectomía radical y no se planteó la opción de quimioterapia.

\section{Seguimiento y Resultados}

La paciente posterior a la intervención quirúrgica no presenta complicaciones y hasta la presente fecha no ha presentado deterioro en su estado físico a pesar de su avanzada edad 


\section{Discusión}

El pronóstico es relativamente bueno, la mortalidad por esta patología es mínima, la metástasis en los ganglios es de tan solo el 1\%, pues este tipo de tumores tienen un crecimiento muy lento, las recurrencias de este tipo de carcinoma tras la resección local es menos del $5 \%$.

Las lesiones papilares de la mama comparten características clínicas entre sí el diagnóstico diferencial se lo realiza dentro del punto de vista citológico:

El papiloma intraductal es el grupo más frecuente de las lesiones papilares de la mama afectando hasta a un $5,3 \%$ de la población. Pueden ser papilomas centrales (retroareolares) o encontrarse en la periferie y se presentan como lesiones arborescentes centradas por un eje fibrovascular y revestidas por una capa de células mioepiteliales sobre las que se encuentra otra proliferación de células epiteliales [12]

El carcinoma papilar encapsulado. Se caracteriza por poseer ejes fibrovasculares habitualmente finos, revestidos por una población monomorfa de células con escasa o moderada atipia. Se diferencia del carcinoma papilar intraductal porque no posee células mioepiteliales no solo en el seno de las papilas sino también en la periferia de la lesión.

El carcinoma papilar sólido, está compuesto por nidos sólidos, con finos ejes fibrovasculares en su interior que son difíciles de apreciar [12]. No es infrecuente la ausencia de celulas una fina capa de celulas epiteleales periféricas.

El papiloma atípico es aquella lesión papilar que no posee todas las características histológicas del CDIS.

El carcinoma papilar intraductal, es raro pues se representa el 1 por ciento de los carcinomas mamarios, representa aproximadamente el 0,5-1\% de todos los carcinomas de mama [12]. Se presenta en mujeres posmenopáusicas, con una edad promedio entre 64 y 67 años, pueden ser solitarios o múltiples, se localizan en la región retro alveolar o subalveolar como una masa solitaria de bordes bien definidos, aunque en ocasiones las mujeres pueden presentar telorragia en alrededor del 20 al 35 por ciento de los casos [7]. Pueden manifestarse como tumores bien circunscritos sólidos los mismos que se presentan como multilobulados y quísticos que son uni o multiloculares, en el centro del quiste se observa una luz que contiene tejido necrótico rodeado de tejido hemorrágico [13]. El carcinoma papilar intraquístico, usualmente se origina en mujeres de edad avanzada $[14,15]$.

El estudio de [16] describe resultados de la escisión quirúrgica versus la vigilancia por imágenes para mujeres con una lesión mamaria sólida en las imágenes y una lesión papilar benigna en el IGCNB. Es importante destacar que este es el primer estudio busco caracterizar los resultados del cáncer por etapa y factores pronósticos, es mismo es el estudio de cohorte más grande publicado en el que se destaca lesiones masivas benignas de mama papilar con resultados favorables. El estudio de [17] relacionado con el carcinoma papilar intracrástico de mama basado en un análisis de la base de datos SEER de implicaciones para la terapia mostró que la mediana de edad fue de 67 años, por lo que este caso esa implicación respecto a la edad es comparable. 
Se ha demostrado diversos métodos de imagen para su correcto diagnóstico, la mamografía tiene un porcentaje de sensibilidad del 35 al 40\%, en la que se observa una masa redonda u ovalada de márgenes circunscritos. La ecografía tiene una sensibilidad del 80 al 85 por ciento se presentan como lesiones hipo o hiperecogénicas en ocasiones es difícil diferenciar la lesión papilar de secreciones espesas intraductales, se presentan como masas complejas y sólidas [18].

En la galactografía se observa una obstrucción ductal defectos en el llenado y en la pared del ducto se observa irregularidades focales y difusas. En la resonancia magnética nuclear se observa como una masa redonda u ovalada con márgenes bien definidos, internamente se observa como una composición heterogénea, y la presencia de varias masas nodulares de intensidad intermedia que se proyectan desde la periferia a la luz [19]. Lefkowitz y otros informaron de 20 casos de carcinomas papilares intraductales (CPI) de células cuboidales con abundante citoplasma claro o ligeramente eosinófilo. Estas células estaban localizadas principalmente cerca de la membrana del fondo, individualmente, en pequeños grupos o en láminas anchas $[20,21]$. En resumen, las lesiones papilares siguen siendo un campo complicado tanto para el patólogo como para el clínico a la hora de abordar su manejo. La tendencia a realizar procedimientos diagnósticos cada vez menos invasivos aumenta el número de estas lesiones diagnosticadas mediante citología o punción con aguja. En estos casos, ante la falta de criterios histológicos inequívocos que permitan diferenciar claramente cada entidad, el uso de marcadores que identifiquen las células mioepiteliales puede resultar de gran ayuda [22, 23]. Por lo general, es difícil diferenciar entre las lesiones in situ y las invasivas y en la biopsia de núcleo, ya que la invasión suele reconocerse en la periferia de la lesión [24, 25].

\section{Perspectiva del Paciente}

La paciente a pesar de su avanzada edad se siente optimista pues se informó que patología que presenta tiene recurrencia sea mínima y que la metástasis sea nula.

\section{Agradecimiento}

A la paciente por permitirnos presentar su caso clínico y a los profesionales de salud que nos facilitaron la recopilación de la información.

\section{References}

[1] Martínez J, Rodríguez M, Martínez V, Fumero L. Carcinoma papilar invasor de la mama. Presentación de un caso. Medisur. 2017;15(4):550-6.

[2] Esposito E, Di Bonito M, lodice G, et al. Intracystic papillary breast carcinoma with DCIS in a man: a case report. Transl Cancer Res. 2019;8(S5):S445-8.

[3] Pérez JA, Soto S. Análisis del período 1998-2002 en el Hospital Clínico Regional de Valdivia. Cuad Cir. 2003;(17):23-9.

[4] Pérez PJA, Mariángel PP, Poblete SMT. Carcinoma mamario papilar intraquístico. Cuad Cirugía. 2004;18(1):70-5. 
[5] Fakhreddine $\mathrm{MH}$, Haque W, Ahmed A, et al. Prognostic factors, treatment, and outcomes in early stage, invasive papillary breast cancer. Am J Clin Oncol Cancer Clin Trials .2018;41(6):532-7.

[6] Guo S, Wang Y, Rohr J, et al. Solid papillary carcinoma of the breast: A special entity needs to be distinguished from conventional invasive carcinoma avoiding over-treatment. Breast. 2016;26:67-72.

[7] Rocha-Rodríguez MG, Álvarez-Alfonso BY, Casián-Castellanos GA, Rodríguez-Blas Al. Carcinoma papilar: reporte de un caso y revisión de la literatura. Rev Hosp Jua Mex. 2014;81(2):126-30.

[8] Kumar S, Lau S, Kruper L, et al. Papillary carcinoma of the breast: An overview. Breast Cancer Res Treat. 2010;122(3):637-45.

[9] Calderón NJM, Febles G. Actualidad para el diagnóstico del carcinoma ductal in situ: Correlación radiopatológica. Horiz Médico. 2017;17(1):57-65.

[10] Salles MDA, Matias MARF, Perez AA, Gobbi H. Carcinoma ductal in situ da mama: Critérios para diagnóstico e abordagem em hospitais públicos de Belo Horizonte. Rev Bras Ginecol e Obstet. 2006;28(12):721-7.

[11] Martínez A, Aparicio I, Hernández G, Muñoz MDM, De Santiago J. Factores pronósticos del carcinoma ductal in situ de mama. Rev Chil Obstet Ginecol. 2008;73(1):35-41.

[12] Mok CW, Lee WP, Ng WLV, Tan S-M. Intracystic papillary breast carcinoma in a male patient: a case report and review of literature. J Surg Case Reports. 2018;2018(11):1-4.

[13] Imtiaz S, Irshad M, Usman R, Saad M. Synchronous bilateral intracystic papillary and invasive ductal breast carcinoma in a young female: a rare entity. Sri Lanka J Radiol. 2018;3(1):5.

[14] González OJ, González DA, Díaz VM, Fleites AA, Roque LJ, Dueñas GD. Carcinoma papilar intraquístico de la mama. Reporte de un caso. Rev Médica Electrónica. 2016;38(2):270-6.

[15] Markopoulos C, Kouskos E, Gogas H, et al. Diagnosis and treatment of intracystic breast carcinomas. Am Surg. 2002;68(9):783-6.

[16] Kuehner G, Darbinian J, Habel L, et al. Benign papillary breast mass lesions: Favorable outcomes with surgical excision or imaging surveillance. Ann Surg Oncol. 2019;26(6):1695- 703.

[17] Mogal H, Brown DR, Isom S, Griffith K, Howard-McNatt M. Intracystic papillary carcinoma of the breast: A SEER database analysis of implications for therapy. Breast. 2016;27:87-92.

[18] Martínez de Vega V, Linares S, Alvarez L, Recio M, Daimiel I. Comportamiento en Resonancia Magnética de mama del variado espectro de lesiones papilares de la mama. Soc Española Radiol Médica. 2018;130.

[19] Sarmiento-Martínez HI, Ramírez-Peña JL, Salas-Longoria JM, Ovalle-Escalera CN, Martínez-García E. Carcinoma papilar intraquístico de mama en hombre. Rev An Radiol México. 2019;18(1):52-8.

[20] Ueno N, Kuroda H, Kozuka Y, et al. Dimorphic variant of ductal carcinoma in situ of the breast. Hum Pathol Case Reports 2017;10:92-5.

[21] Ueno N, Kuroda $\mathrm{H}$, Kurosumi M, et al. Clinicopathological study of a dimorphic variant of breast carcinoma. Breast Cancer. 2018;25(2):151-8.

[22] Peg V. Lesiones papilares de la mama Papillary lesions of the breast. Rev Senol Patol Mamar. 2013;26(2):39-40.

[23] Sarica O, Dokdok M. Imaging findings in papillary breast lesions: An analysis of ductal findings on magnetic resonance imaging and ultrasound. J Comput Assist Tomogr. 2018;42(4):542-51.

[24] Ingle SB, Murdeshwar H, Siddiqui S. Papillary carcinoma of breast: Minireview. World J Clin Cases. 2016;4(1):20-4.

[25] Fernandez LL. Características Clínico Patológicas Del Cáncer De Mama En Mujeres Menores De Cincuenta Años, Hospital Nacional Guillermo Almenara Irigoyen, 2009 - 2010. Rev Medica Carrionica [Internet]. 2016;3(1):2009-10. Disponible en: http://cuerpomedico.hdosdemayo.gob.pe/ index.php/revistamedicacarrionica/article/view/31/25 